\title{
EFFECTS OF PROPYLENE GLYCOL AND MAGNESIUM CHLORIDE AGAINST DERMATOPHYTES ISOLATED FROM COMPANION ANIMALS
}

\author{
A. D. Singh, C. Debnath, A. BAnerJeE ${ }^{1}$, K. BATAByal ${ }^{1}$ \\ B. ROY ${ }^{2}$ AND I. SAMANTA*1 \\ Department of Veterinary Public Health \\ Faculty of Veterinary and Animal Sciences \\ West Bengal University of Animal and Fishery Sciences \\ 37, K. B. Sarani, Kolkata-700 037, W.B., India
}

\begin{abstract}
The present study was undertaken to detect the occurrence of different dermatophytes in clinically suspected dogs and cats, and to evaluate the antifungal activities of propylene glycol and magnesium chloride against the isolates. A total of 517 samples including skin scrapings, hair, and nail scrapings were collected from suspected dogs $(n=428)$ and cats $(n=89)$ in West Bengal, India. Standard mycological technique was followed for isolation and identification of dermatophytes. Microsporum canis was isolated with highest frequency from both dogs and cats followed by Trichophyton mentagrophytes and Microsporum gypseum. Minimum inhibitory concentration of propylene glycol against selected isolates of M. canis, M. gypseum and T. mentagrophytes was detected as $0.008 \mathrm{~g} / \mathrm{L}$, whereas, magnesium chloride produced a minimum inhibitory concentration of $0.016 \mathrm{~g} / \mathrm{L}$ against the same isolates. The study confirmed antifungal effect of both propylene glycol and magnesium chloride against studied dermatophyte isolates and better inhibitory property of propylene glycol.
\end{abstract}

Key words: Antifungal, Dermatophyte, Magnesium chloride, Propylene glycol

Dermatophytes are aerobic fungal pathogens which are able to damage and utilize keratin for their growth as a nutrient substrate found in keratinized layers of skin, hair and nails. They are divided into anthropophilic (infect humans and rarely animals), zoophilic species (affects animals and are capable of infecting people) and

\footnotetext{
*Corresponding author

${ }^{1}$ Department of Veterinary Microbiology, Faculty of Veterinary and Animal Sciences, WBUAFS, 37, K.B. Sarani, Kolkata-700 037, W.B

${ }^{2}$ Department of Animal Nutrition, Faculty of Veterinary and Animal Sciences, WBUAFS, 37, K.B. Sarani, Kolkata-700 037, W.B
} 
geophilic species (can infect both animals and humans) (Samanta, 2015). Clinical signs of alopecia, pruritis shown by dogs and cats are because of hair follicle damage and inflammation. The main etiological agents isolated from dogs and cats are Microsporum canis, Trichophyton mentagrophytes and Microsporum gypseum (Lewis et al., 1991; Sparkes et al., 1993).

Dermatophytes can be treated both topically and systemically. While systemic treatment with terbinafine or itraconazole for onychomycosis and other severe and chronic dermatophyte infections may be helpful, but adverse effects such as gastrointestinal complications, rashes and severe hepatotoxicity cannot be ignored (De Berker, 2009). Recent emergence of resistant clinical isolates leads to failure in the treatment of dermatophytoses (AlcazarFuoli and Mellado, 2014). Propylene glycol has proven antibacterial, keratolytic and antifungal activities (Kinnunen and Koskel, 1991). Topical application of propylene glycol along with urea and lactic acid had earlier shown antifungal activity (Emtestam et al., 2012). Magnesium chloride was also detected to possess growth inhibitory effects on dermatophytes at a low concentration (Okafor and Ngwogu, 1999). However, studies on antifungal activities of propylene glycol and magnesium chloride against dermatophytes isolated from clinically suspected animals are still scanty. The present study was undertaken to detect the occurrence of different dermatophytes in clinically suspected dogs and cats and to evaluate the antifungal activities of propylene glycol and magnesium chloride against the isolates.

\section{MATERIALS AND METHODS}

Sampling: A total of 517 samples including skin scrapings, hair, and nail scrapings were collected from suspected dogs $(n=428)$ and cats $(n=89)$ showing signs of dermatophytoses such as alopecia, annular plaques, scaling, crusts and desquamation of paws from a metropolitan city (Kolkata) in West Bengal, India (Fig. 1, Fig. 2, Fig. 3).

Isolation and identification of dermatophytes: The collected samples were inoculated into dermatophyte test medium (HiMedia ${ }^{\circledR}$, India) followed by Sabouraud's dextrose agar (SAD) (HiMedia $^{\circledR}$, India) for growth. The growth on SDA was identified by colony characteristics, hyphal and conidial cells. Conidia were stained with lactophenol cotton blue (HiMedia ${ }^{\circledR}$, India) and were identified on the basis of their shape, size, presence of septa, thickness of conidial wall and arrangement of conidial cells around the hyphae (Pang et al., 2008). Further, the isolates were confirmed by rice grain test, hair perforation test, urease test, temperature tolerance test, pigment production and growth on trichophyton agar (Samanta, 2015).

Antifungal sensitivity testing: Antifungal sensitivity of propylene glycol (West Bengal Chemical Industries Limited ${ }^{\circledR}$, India) and magnesium chloride (West 
Bengal Chemical Industries Limited ${ }^{\circledR}$, India) against all dermatophyte isolates was performed using broth micro-dilution assay (National Committee for Clinical Laboratory Standards, 2002). All the isolates were grown on oatmeal cereal agar (HiMedia ${ }^{\circledR}$, India) at $25 \pm 3^{\circ} \mathrm{C}$ for 7 to 10 days. Sterile normal saline $(85 \%)$ was added to the slant, and the culture was gently scrubbed with a sterile loop to dislodge the conidia from the hyphal mat. The optical density of the supernatant containing conidia and fine hyphal fragments was read by double beamed UV spectrophotometer at $530 \mathrm{~nm}$, adjusted to transmittance of $60-70 \%\left(2-4 \times 10^{6}\right.$ cells/ $\mathrm{mL}$ ) and diluted with RPMI-1640 medium to obtain the final inoculums concentration of approximately $0.4-5 \times 10^{4}$ cells $/ \mathrm{mL}$. The test drugs (PG and $\mathrm{MC}$ ) were prepared as stock solution of $1 \mathrm{mg} / \mathrm{mL}$. RPMI-1640 with L-glutamine but without sodium bicarbonate and buffered at $\mathrm{pH} 7.0$ (HiMedia ${ }^{\circledR}$, India) was the medium used for broth microdilution assay. Serial two fold dilutions of the test drugs were prepared and the final concentration ranged from 64.0 to $0.13 \mu \mathrm{g} / \mathrm{mL}$. The micro dilution plates (Tarson ${ }^{\circledR}$, India) were incubated at $25 \pm 3^{\circ} \mathrm{C}$ and were read visually after $3-4$ days of incubation. Endpoint determination values were performed visually in every 24 hours until the indication of growth in positive growth control well.

\section{RESULTS}

The present study isolated Microsporum canis (89/428, 20.7\%), Trichophyton mentagrophytes $(81 / 428,18.9 \%)$ and Microsporum gypseum (13/428, 3.03\%) from the collected samples of dogs suspected for clinical dermatophytoses (Fig. $4,5,6)$. Similarly, from the cats suspected for clinical dermatophytoses, Microsporum canis (15/89, 16.8\%), Trichophyton mentagrophytes $(6 / 89,6.7 \%)$ and Microsporum gypseum (3/89, 3.3\%) were isolated.

Antifungal sensitivity of propylene glycol and magnesium chloride against all the dermatophyte isolates was performed using broth micro-dilution assay. Minimum inhibitory concentration (MIC) of propylene glycol against the isolates of $M$. canis, $M$. gypseum and T. mentagrophytes was detected as $0.008 \mathrm{~g} / \mathrm{L}$, whereas, magnesium chloride produced a MIC of $0.016 \mathrm{~g} / \mathrm{L}$ against the same isolates.

\section{DISCUSSION}

The present study isolated Microsporum canis (89/428, 20.7\%), Trichophyton mentagrophytes $(81 / 428,18.9 \%)$ and Microsporum gypseum (13/428, 3.03\%) from the collected samples of dogs suspected for clinical dermatophytoses. In a prevalence study in Italy, M. canis constituted majority (83\% and $97 \%$ from dogs and cats, respectively) of the isolated dermatophytes followed by $M$. gypseum (13\% and $2.6 \%$ ) and T. mentagrophytes (5.5\% and $0.2 \%$ ) (Mancianti et al., 2003). Previous study with healthy dogs and cats also revealed occurrence of $M$. canis (43.55\%), M. gypseum (36.69\%) and $T$. 
Indian Journal of Animal Health, December, 2018

Antifungal activity against dermatophytes

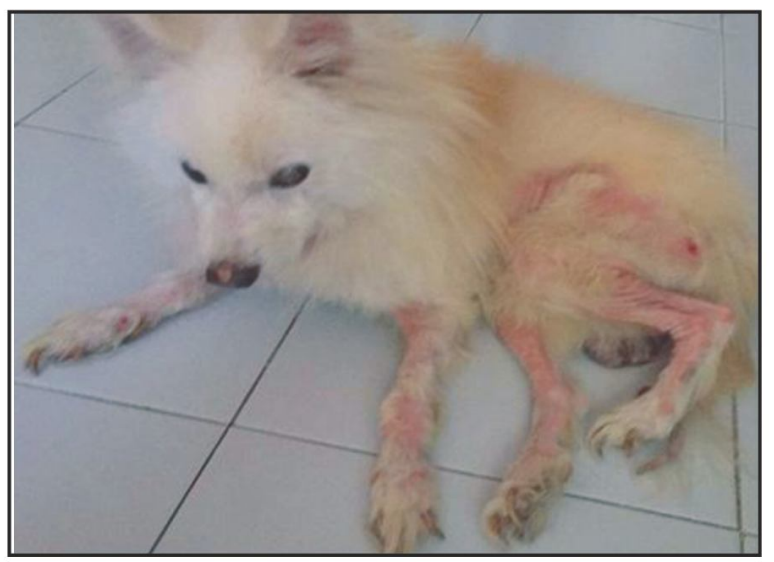

Fig. 1. Alopecia and skin lesions in a dermatophytosis suspected dog

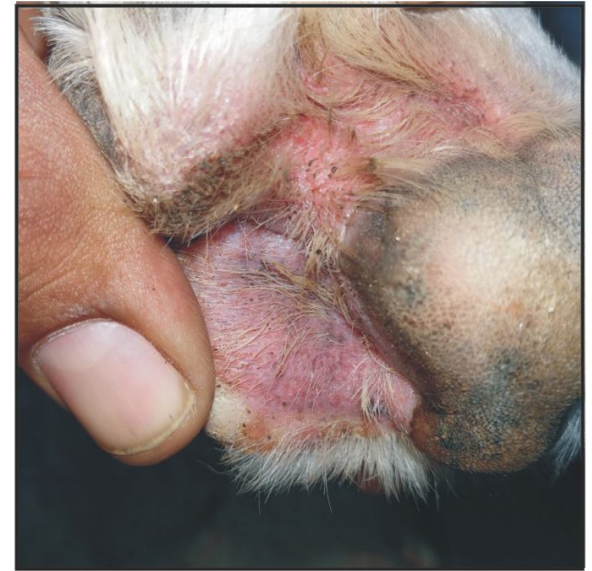

Fig. 2. Desquamation of paws in a dermatophytosis suspected dog

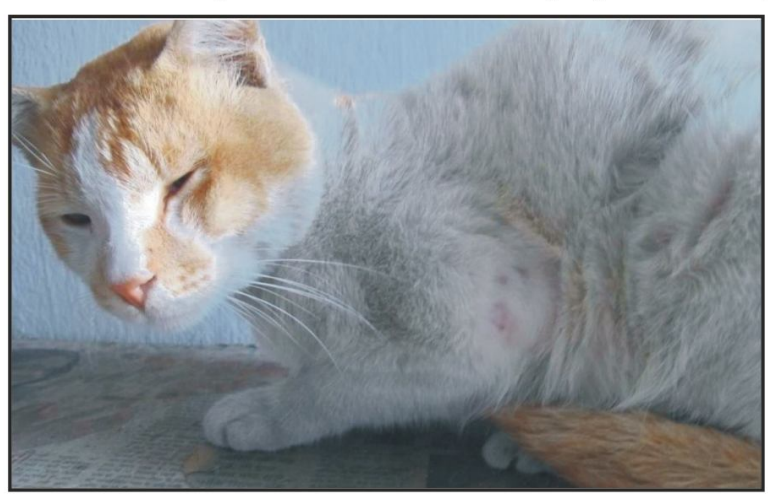

Fig. 3. Alopecia in a dermatophytosis suspected cat 
Indian Journal of Animal Health, December, 2018

Antifungal activity against dermatophytes

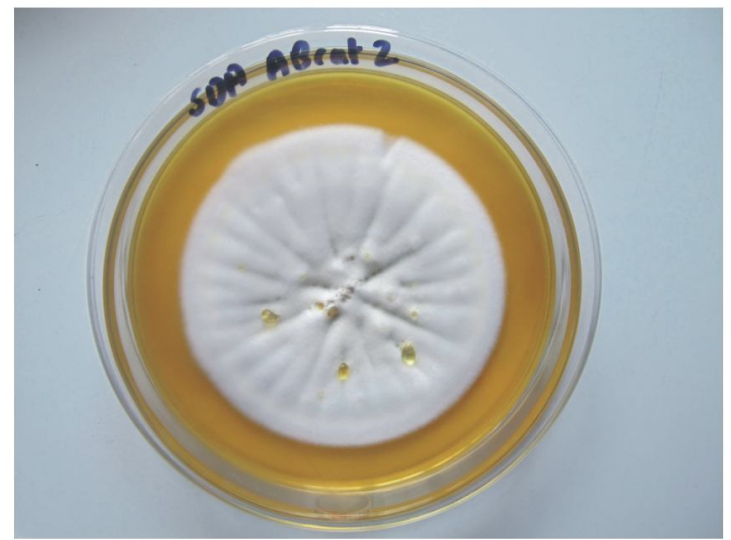

Fig. 4. Microsporum canis colony in SDA plate

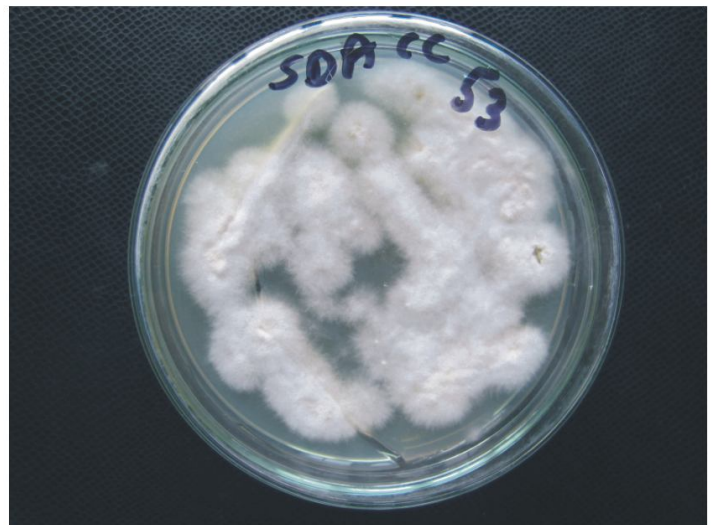

Fig. 5. Microsporum gypseum colony in SDA plate

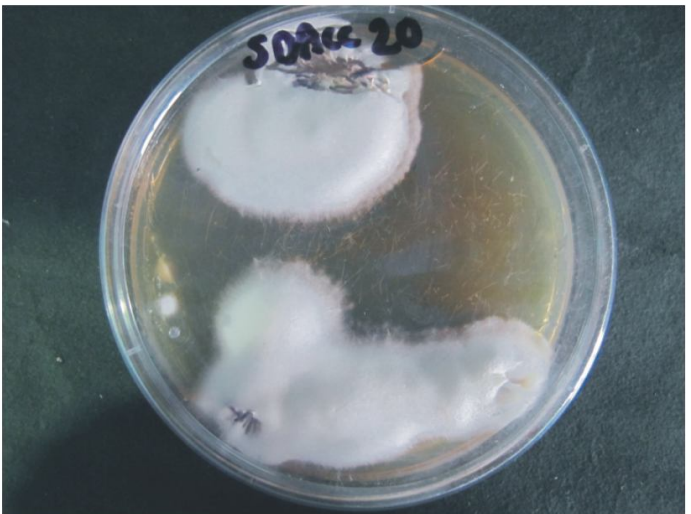

Fig. 6. Trichophyton mentagophytes colony in SDA plate 
mentagrophytes (19.79\%) (Debnath et al., 2016). Occurrence rate of dermatophytes varies with breeds of the companion animals, geographical location, season, and moreover, maintenance of overall hygienic conditions (Samanta, 2015). Detection of M. canis as predominant dermatophyte in companion animals is corroborative with earlier studies reported from India and throughout the world (Seker and Dogan, 2011; da Costa et al., 2013; Beigh et al., 2014).

Due to indiscriminate use and easy availability of antifungals such as azoles and allyl-amines, antifungal resistance is a global concern now a day (Martinez-Rossi et al., 2008). The present study was conducted to detect MIC of propylene glycol and magnesium chloride against selected dermatophyte isolates as an alternative to traditional antifungals. Minimum inhibitory concentration (MIC) of propylene glycol against selected isolates of $M$. canis, M. gypseum and $T$. mentagrophytes was detected as 0.008 $\mathrm{g} / \mathrm{L}$, whereas, magnesium chloride produced a MIC of $0.016 \mathrm{~g} / \mathrm{L}$ against the same isolates. Earlier study indicated MIC of propylene glycol as 30-90 g/L against $T$. mentagrophyte var. interdigitale, T. rubrum and Epidermophyton floccosum (Faergemann and Fredriksson, 1980).

\section{REFERENCES}

Alcazar-Fuoli L and Mellado E, 2014. Current status of antifungal resistance and its
Magnesium chloride at a concentration between $0.1(\mathrm{M})$ and $1(\mathrm{M})$ showed less than $50 \%$ inhibitory effect on T. rubrum, $M$. audouinii, T. mentagrophytes, T. tonsurans and M. gypseum (Okafor and Ngwogu, 1999). Variation in MIC value against fungal isolates occurred with strains, measurement protocol, duration of measurement, density of starting culture, and induction of resistance (Schuurmans et al., 2009; Terwee et al., 2010). Present study also confirmed better antifungal effect of propylene glycol than magnesium chloride on the studied dermatophyte isolates. Such kind of comparative antifungal study is apparently not available to validate the finding.

The present study confirmed the occurrence of $M$. canis as predominant dermatophyte in companion animals and detected MIC values of propylene glycol and magnesium chloride against selected dermatophyte isolates.

\section{ACKNOWLEDGEMENT}

The authors express sincere gratitude to the Hon'ble Vice Chancellor, West Bengal University of Animal and Fishery Sciences for providing infrastructure and Indian Council of Agricultural Research (ICAR) for the necessary funds under 'Outreach programme on zoonotic diseases'.

impact on clinical practice. $\mathrm{Br} \mathrm{J}$ Haematol, 166: 471-484 
Beigh SA, Soodan JS, Singh R, Khan AM and Dar MA, 2014. Evaluation of trace elements, oxidant/antioxidant status, vitamin $C$ and $\beta$ carotene in dogs with dermatophytosis. Mycoses, 57: 358-365

da Costa FV, Farias MR, Bier D, de Andrade CP and de Castro LA et al., 2013. Genetic variability in Microsporum canis isolated from cats, dogs and humans in Brazil. Mycoses, 56: 582-588

De Berker D, 2009. Fungal nail disease. Nw Eng J Med, 360: 2108-2116

Debnath C, Mitra T, Kumar A and Samanta I, 2016. Detection of dermatophytes in healthy companion dogs and cats in eastern India. Iranian J Vet Res, 17: 20

Emtestam L, Kaaman T and Rensfeldt K, 2012. Treatment of distal subungual onychomycosis with a topical preparation of urea, propylene glycol and lactic acid: results of a 24 week, double blind, placebo controlled study. Mycoses, 55: 532-540

Faergemann J and Fredriksson T, 1980. Antimycotic activity of propane-1, 2-diol (propylene glycol). Sabouraudia, 18: 163-166

Kinnunen T and Koskel M, 1991. Antibacterial and antifungal properties of propylene glycol, hexylene glycol, and 1,3-butylene glycol in vitro. Acta Dermto Venero, 71: 148-150
Lewis DT, Foil CS and Hosgood G, 1991. Epidemiology and clinical features of dermatophytosis in dogs and cats at Louisiana State University: 1981-1990. Vet Dermatol, 2: 53-58

Mancianti F, Nardoni S, Cecchi S, Corazza M and Taccini F, 2003. Dermatophytes isolated from symptomatic dogs and cats in Tuscany, Italy during a 15-yearperiod. Mycopathol, 156: 13-18

Martinez-Rossi NM, Peres NT and Rossi A, 2008. Antifungal resistance mechanisms in dermatophytes. Mycopathol, 166: 369

National Committee for Clinical Laboratory Standards, 2002. Reference method for broth dilution antifungal susceptibility testing of filamentous fungi. Approved standard M38-A. National Committee for Clinical Laboratory Standards, Wayne, $\mathrm{Pa}$

Okafor JI and Ngwogu A, 1999. In vitro effects of three metallic salts and soot on the growth of five species of the dermatophytes. J Commun Dis, 31: 165168

Pang SH, Ren P and Wu XJ, 2008. Diagnosis and treatment of rabbit skin mildew. Anim Sci Vet Med, 4: 70

Samanta I, 2015. Veterinary Mycology. $1^{\text {st }}$ Edn., Springer

Schuurmans JM, Hayali ASN, Koenders BB 
and ter Kuile BH, 2009. Variations in MIC value caused by differences in experimental protocol. J Microbiol Methods, 79: 44-47

Seker E and Dogan N, 2011. Isolation of dermatophytes from dogs and cats with suspected dermatophytosis in Western Turkey. Prev Vet Med, 98: 46-51

Sparkes AH, Gruffydd-Jones TJ, Shaw SE,
Wright AI and Stokes CR, 1993. Epidemiological and diagnostic features of canine and feline dermatophytosis in the United Kingdom from 1956 to 1991. Vet Rec, 133: 57-61

Terwee CB, Roorda LD, Dekker J, BiermaZeinstra SM, and Peat G et al., 2010. Mind the MIC: large variation among populations and methods. J Clin Epidemiol, 63: 524-534 